\title{
Posterior cerebral artery stenosis and posterior circulation revascularization surgery in pediatric patients with moyamoya disease
}

\author{
Tomomi Kimiwada, MD, PhD, ${ }^{1}$ Toshiaki Hayashi, MD, PhD, ${ }^{2}$ Reizo Shirane, MD, PhD, ${ }^{1}$ and \\ Teiji Tominaga, MD, PhD ${ }^{3}$
} ${ }^{1}$ Department of Neurosurgery, Miyagi Children's Hospital; ${ }^{2}$ Department of Neurosurgery, Sendai City Hospital; and ${ }^{3}$ Department
of Neurosurgery, Tohoku University Graduate School of Medicine, Sendai, Japan

\begin{abstract}
OBJECTIVE Some pediatric patients with moyamoya disease (MMD) present with posterior cerebral artery (PCA) stenosis before and after anterior circulation revascularization surgery and require posterior circulation revascularization surgery. This study evaluated the factors associated with PCA stenosis and assessed the efficacy of posterior circulation revascularization surgery, including occipital artery (OA)-PCA bypass, in pediatric patients with MMD.
\end{abstract}

METHODS The presence of PCA stenosis before and after anterior circulation revascularization surgery and its clinical characteristics were investigated in 62 pediatric patients ( $<16$ years of age) with MMD.

RESULTS Twenty-three pediatric patients (37\%) with MMD presented with PCA stenosis at the time of the initial diagnosis. A strong correlation between the presence of infarction and PCA stenosis before anterior revascularization was observed ( $p$ <.001). In addition, progressive PCA stenosis was observed in 12 patients $(19.4 \%)$ after anterior revascularization. The presence of infarction and a younger age at the time of initial diagnosis were risk factors for progressive PCA stenosis after anterior revascularization ( $p<0.001$ and $p=0.002$, respectively). Posterior circulation revascularization surgery, including OA-PCA bypass, was performed in 9 of the 12 patients with progressive PCA stenosis, all of whom showed symptomatic and/or radiological improvement.

CONCLUSIONS PCA stenosis is an important clinical factor related to poor prognosis in pediatric MMD. One should be aware of the possibility of progressive PCA stenosis during the postoperative follow-up period and consider performing posterior circulation revascularization surgery.

https://thejns.org/doi/abs/10.3171/2018.1.PEDS17367

KEYWORDS moyamoya disease; posterior cerebral artery; posterior circulation revascularization surgery; vascular disorders

$\mathrm{M}$ oyamoya disease (MMD) is characterized by progressive stenosis or occlusion of the terminal portions of the bilateral internal carotid arteries (ICAs) or proximal anterior and middle cerebral artery (ACA/MCA) with abnormal collateral vessel formation. ${ }^{26}$ In pediatric patients with MMD, the initial clinical manifestations at diagnosis include transient ischemic attacks (TIAs) and cerebral infarctions, and this population shows a more progressive clinical course than their adult counterparts. Various revascularization surgeries have been shown to improve cerebral blood flow $(\mathrm{CBF})$ and prevent cerebral infarction. ${ }^{8,914-16}$ However, some patients with MMD present with stenoocclusive posterior cerebral artery (PCA) lesions, ${ }^{20}$ followed by cerebral infarction after anterior circulation revascularization surgery. Recent studies emphasized PCA involvement in MMD as an important factor related to poor prognosis. ${ }^{4-7,12,22-24}$ However, few reports have detailed posterior circulation revascularization surgery. ${ }^{3,12,18}$

The current study reviewed 62 pediatric patients with

ABBREVIATIONS ACA = anterior cerebral artery; $\mathrm{CBF}=$ cerebral blood flow; $\mathrm{CPP}=$ cerebral perfusion pressure; $\mathrm{CVR}=$ cerebrovascular reserve; $\mathrm{EDMS}=$ encephaloduromyosynangiosis; ICA = internal carotid artery; ${ }^{123}$-IMP = N-isopropyl- $p$-[123] $]$ iodoamphetamine; $M C A=$ middle cerebral artery; $M M D=$ moyamoya disease; $M R A=$ MR angiography; $\mathrm{OA}=$ occipital artery; $\mathrm{PCA}=$ posterior cerebral artery; STA = superficial temporal artery; TIA = transient ischemic attack.

SUBMITTED July 2, 2017. ACCEPTED January 2, 2018.

INCLUDE WHEN CITING Published online April 6, 2018; DOI: 10.3171/2018.1.PEDS17367. 
MMD and retrospectively assessed PCA involvement and the efficacy of posterior circulation revascularization surgery, including occipital artery (OA)-PCA anastomosis.

\section{Methods \\ Patient Population}

We retrospectively analyzed cases of patients with MMD who underwent surgery between April 2004 and January 2015 in the Department of Neurosurgery at Miyagi Children's Hospital; 62 consecutive pediatric patients (age $<16$ years) were included in this study. Patients with a follow-up period of $<1$ year were excluded. A definitive diagnosis was provided using MRI and MR angiography (MRA) following the guidelines of the Research Committee on MMD by the Japanese Ministry of Health and Welfare. $^{25}$

\section{Data Collection}

We recorded age, sex, clinical symptoms at onset, family history of MMD, PCA stenosis at onset, progression of PCA stenosis, and posterior revascularization after anterior revascularization for each patient. Preoperatively, all 62 patients underwent MRI using diffusion-weighted, T2weighted, and FLAIR images; MRA; and qualitative basal $N$-isopropyl- $p$-[123I] iodoamphetamine ( ${ }^{123}$ I-IMP) SPECT. Qualitative acetazolamide-stressed $(20 \mathrm{mg} / \mathrm{kg}$; maximum dose $1 \mathrm{~g}$ ) ${ }^{123}$ I-IMP-SPECT was performed in patients except for those with frequent transient neurological deficits.

Postoperative MRI and MRA were performed on postoperative day 7, at 4-6 months, and annually thereafter. Postoperative ${ }^{123}$ I-IMP-SPECT was performed on postoperative day 3 or 4 and at 4-6 months after surgery in all cases. The radiological findings were assessed by radiologists who were blinded to the patients' clinical course. Follow-up data were obtained from clinical charts or by telephone communication.

\section{Surgical Treatment}

Our surgical indications for patients with MMD were as follows: ischemic symptoms or decreased CBF and/or cerebrovascular reserve (CVR) as measured by ${ }^{123}$ I-IMPSPECT. All patients were undergoing their first surgery and had no history of microsurgical treatment. As a firstline treatment, we performed combined bypass surgery for the anterior circulation, consisting of superficial temporal artery (STA) to MCA anastomosis with indirect pial synangiosis. Additional posterior revascularization surgery was indicated for patients with 1) progressive PCA stenosis on MRA after anterior revascularization; 2) recurrent cerebral ischemic manifestations, such as TIAs or cerebral infarctions, attributable to PCA and/or ACA lesions; and/or 3) progressive decreases in $\mathrm{CBF}$ and/or CVR of the posterior circulation during the follow-up periods after anterior revascularization. Because it is especially difficult in young children to evaluate whether the patient has symptoms due to the PCA stenosis, we performed posterior revascularization surgery even for asymptomatic patients who showed progressive PCA stenosis and decreased $\mathrm{CBF}$ and/or CVR in the posterior circulation by ${ }^{123}$ I-IMP-SPECT.

\section{Statistical Analysis}

The statistical analysis was performed using JMP software (version 11, SAS Institute, Inc.). Fisher's exact tests were used to compare each categorical variable between the 2 groups, including sex, clinical symptoms at onset, family history of MMD, progression of PCA stenosis, and posterior revascularization after anterior revascularization. Welch's t-tests were used to compare continuous variables, such as age at onset; $p$ values $<0.05$ were considered statistically significant.

\section{Results \\ Patient Characteristics}

A total of 62 consecutive pediatric patients with MMD (27 males, 35 females; median age 7.6 years, range 6 months to 15 years) were included, and no patients were excluded due to a lack of follow-up. Familial MMD was observed in 15 (24\%) patients. The clinical symptoms at onset included cerebral infarctions in 24 patients (38.7\%), TIA in 21 (33.9\%), headache in $8(12.9 \%)$, cerebral hemorrhage in $4(6.5 \%)$, seizures in $3(4.8 \%)$, and other in 2 (3.2\%; Table 1).

\section{PCA Stenosis at Onset}

Twenty-three (37\%) of the 62 patients presented with PCA stenosis at the time of the initial MRA. There were no significant differences in age, sex, family history, or PCA stenosis at onset between patients with and without PCA stenosis. Among the 24 patients with cerebral infarction at onset, $16(66.7 \%)$ showed PCA stenosis at the initial MRA. There was a strong correlation between the presence of infarction and PCA stenosis at onset $(66.7 \%$ vs $20.5 \%$, respectively, $\mathrm{p}<0.001$; Table 1 ).

\section{Progression of PCA Stenosis After Anterior Revascularization}

Progressive PCA stenosis was observed in 12 (19.4\%) of 62 patients following anterior revascularization during the follow-up period. Three predictive factors were associated with progressive PCA stenosis after anterior revascularization. First, younger age was statistically correlated with progressive PCA stenosis in pediatric patients with MMD after anterior revascularization (mean age 5.1 vs 8.2 years, $p<0.002$ ). Second, among the 12 patients with progressive PCA stenosis, 10 (83.3\%) showed cerebral infarction as their initial symptom. The presence of infarction at the time of initial diagnosis was a risk factor for progressive PCA stenosis after anterior revascularization (83.3\% vs $28.0 \%, \mathrm{p}<0.001$; Table 2 ). And third, 8 (34.8\%) of 23 patients with PCA stenosis at onset showed progressive PCA stenosis after anterior revascularization. The presence of stenoocclusive lesions involving the PCA on initial MRA was significantly associated with progressive PCA stenosis after anterior revascularization (34.8\% vs $10.3 \%, \mathrm{p}=0.042$; Table 1 ).

\section{Posterior Revascularization Surgery}

We performed posterior circulation revascularization surgery in 9 of the 12 patients with progressive PCA ste- 
TABLE 1. Clinical characteristics of patients with and without PCA stenosis at onset

\begin{tabular}{lccc}
\hline \multicolumn{1}{c}{ Characteristic } & $\begin{array}{c}\text { PCA } \\
\text { Stenosis } \\
(\mathrm{n}=23)\end{array}$ & $\begin{array}{c}\text { No PCA } \\
\text { Stenosis } \\
(\mathrm{n}=39)\end{array}$ & $\begin{array}{c}\mathrm{p} \\
\text { Value }\end{array}$ \\
\hline Mean age at onset \pm SD $(\mathrm{yrs})$ & $7.2 \pm 4.4$ & $7.8 \pm 3.7$ & 0.552 \\
\hline Females & 15 & 20 & 0.305 \\
\hline Family history of MMD & 4 & 11 & 0.378 \\
\hline Symptom at onset & & & \\
\hline TIA & 4 & 17 & 0.052 \\
\hline Infarction & 16 & 8 & $<0.001$ \\
\hline Headache & 1 & 7 & 0.239 \\
\hline Hemorrhage & 0 & 4 & 0.287 \\
\hline Seizure & 0 & 3 & 0.289 \\
\hline Other & 2 & 0 & \\
\hline Progression of PCA stenosis & 8 & 4 & 0.042 \\
\hline Posterior revascularization & 6 & 3 & 0.066 \\
\hline Vas
\end{tabular}

Values are presented as number of patients unless otherwise stated.

nosis after anterior revascularization. The other 3 patients also showed progressive PCA stenosis but demonstrated neither ischemic symptoms nor decreased uptake in ${ }^{123} \mathrm{I}-$ IMP-SPECT. Thus, they did not fulfill our surgical criteria for posterior revascularization and were excluded. Of the 9 patients, 6 (cases 1, 2, 4, 6, 7, and 10; Table 3) showed recurrent TIAs that mainly manifested as lowerextremity weakness, 1 patient (case 5) developed cerebral infarction in the temporooccipital cortex, and 2 patients (cases 3 and 8) were asymptomatic but had progressive decreases in CBF and/or CVR of the posterior circulation during the follow-up period. The posterior revascularization procedures were either OA-PCA anastomoses combined with an indirect revascularization (cases 1-6 and 9 ), or an indirect revascularization only (cases 7 and 8). When we could not find optimal recipient arteries to be anastomosed on the brain surface, we selected indirect revascularization only for these patients (cases 7 and 8). Two patients (cases 3 and 7) underwent bilateral posterior revascularization at one time. Detailed surgical procedures are shown in Table 3. There was no significant difference in age at onset between the 9 patients with posterior revascularization and the 53 patients without (mean ages 5.4 and 7.9 years, respectively). The clinical symptoms at diagnosis were cerebral infarctions in 8 of 9 patients with posterior revascularization. The mean interval between anterior and posterior revascularization surgeries was 2.3 years. Postoperatively, TIAs were eliminated in all 8 patients; however, 1 patient (case 6) experienced acute cerebral infarction in the affected occipital lobe after surgery. Medical treatments such as aspirin were not administered throughout the preoperative and postoperative courses in any of the patients. Table 3 summarizes the characteristics of patients with posterior revascularization.

\section{Illustrative Case}

A 2-year-old girl (case 4) was referred to our depart-
TABLE 2. Clinical characteristics of patients with and without progression of PCA stenosis after anterior revascularization surgery

\begin{tabular}{lccc}
\hline & \multicolumn{2}{c}{ PCA Stenosis } & \\
\cline { 2 - 3 } \multicolumn{1}{c}{ Characteristic } & $\begin{array}{c}\text { Progression } \\
(\mathrm{n}=12)\end{array}$ & $\begin{array}{c}\text { No Progression } \\
(\mathrm{n}=50)\end{array}$ & $\begin{array}{c}\mathrm{p} \\
\text { Value }\end{array}$ \\
\hline Mean age at onset (yrs) \pm SD & $5.1 \pm 2.5$ & $8.2 \pm 4.0$ & 0.002 \\
\hline Females & 7 & 28 & 1.000 \\
\hline Family history of MMD & 3 & 12 & 1.000 \\
\hline Symptom at onset & 1 & 20 & 0.046 \\
\hline TIA & 10 & 14 & $<0.001$ \\
\hline Infarction & 0 & 8 & 0.335 \\
\hline Headache & 0 & 4 & 0.578 \\
\hline Hemorrhage & 1 & 2 & 0.482 \\
\hline Seizure & & & \\
\hline Other & 9 & 0 & $<0.001$ \\
\hline Posterior revascularization & & & \\
\hline
\end{tabular}

Values are presented as number of patients unless otherwise stated.

ment with sudden right hemiparesis and disturbed consciousness. Her aunt was affected with MMD. MRI using diffusion-weighted images showed a high-intensity area in the left frontal lobe, while MRA showed stenosis of the terminal portion of the bilateral intracranial ICA. She was diagnosed as having MMD and cerebral infarction. ${ }^{123}$ IIMP-SPECT at rest showed markedly decreased uptake in the right frontotemporal region (Fig. 1A). Thus, the patient underwent right STA-MCA anastomosis with encephaloduromyosynangiosis (EDMS) in the right hemisphere. Two weeks after the right-sided revascularization, we performed a left STA-MCA anastomosis with EDMS. The postoperative course was uneventful and the patient did not experience any new neurological deficits or TIAs after surgery. The resting ${ }^{123}$ I-IMP-SPECT performed 4 months after the bilateral anterior revascularization showed markedly increased uptake in both hemispheres. MRI studies after surgery demonstrated no additional ischemic lesions (Fig. 1B). The patient's right hemiparesis and disturbed consciousness improved, and she was discharged after the anterior revascularization.

Three years after the bilateral anterior circulation revascularization surgery, she presented with transient bilateral lower limb paresis after playing with soap bubbles. MRI showed no new lesions, but MRA revealed progression of the right PCA stenosis. The ${ }^{123}$ I-IMP-SPECT performed at rest showed markedly decreased uptake in the right PCA territory that was not detected previously (Fig. 1C). Thus, we decided to perform a right OA-PCA anastomosis with indirect revascularization surgery.

\section{OA-PCA Anastomosis With Indirect Revascularization}

The patient was placed prone on a horseshoe-shaped headrest (Fig. 2A). A reverse U-shaped occipital skin incision was made, and the periosteum was left in place on the bone and later dissected to create a periosteal flap (Fig. 2B). The OA was dissected approximately $6 \mathrm{~cm}$ from the 
TABLE 3. Characteristics of patients with posterior revascularization after anterior revascularization

\begin{tabular}{ccclccccc}
\hline $\begin{array}{c}\text { Case } \\
\text { No. }\end{array}$ & $\begin{array}{c}\text { Age at Onset } \\
\text { (yrs), Sex }\end{array}$ & $\begin{array}{c}\text { Initial } \\
\text { Symptom }\end{array}$ & $\begin{array}{c}\text { Recurrent } \\
\text { Symptom }\end{array}$ & $\begin{array}{l}\text { 123/-IMP-SPECT } \\
\text { PR Procedure }\end{array}$ & $\begin{array}{c}\text { Time Between } \\
\text { AR and PR (yrs) }\end{array}$ & $\begin{array}{c}\text { Family } \\
\text { Postop Course }\end{array}$ \\
\hline 1 & $5, \mathrm{~F}$ & Infarction & TIA & Decreased CBF & Rt OA-PCA w/ indirect & 0.88 & TIA disappeared \\
\hline 2 & $5, \mathrm{M}$ & Infarction & TIA & Decreased CBF & Rt OA-PCA w/ indirect & 1 & TIA disappeared & No \\
\hline 3 & $6, \mathrm{~F}$ & Infarction & None & Decreased CVR & Bilat OA-PCA w/ indirect & 0.95 & None & Yes \\
\hline 4 & $2, \mathrm{~F}$ & Infarction & TIA & Decreased CBF & Rt OA-PCA w/ indirect & 3.19 & TIA disappeared & Yes \\
\hline 5 & $3, \mathrm{~F}$ & Epilepsy & Infarction & Decreased CBF & Rt OA-PCA w/ indirect & 4.23 & Hemiparesis & No \\
\hline 6 & $3, \mathrm{M}$ & Infarction & TIA & Decreased CBF & Lt OA-PCA w/ indirect & 2.94 & TIA disappeared, new infarction & No \\
\hline 7 & $7, \mathrm{~F}$ & Infarction & TIA & Decreased CBF & Bilat indirect only & 1.04 & TIA disappeared & No \\
\hline 8 & $8, \mathrm{M}$ & Infarction & None & Decreased CBF & Rt indirect only & 1.17 & None & NIA disappeared \\
\hline 9 & $10, \mathrm{~F}$ & Infarction & TIA & Decreased CBF & Lt OA-PCA w/ indirect & 5.1 & No \\
\hline
\end{tabular}

$\mathrm{AR}=$ anterior revascularization; $\mathrm{PR}=$ posterior revascularization .

skin flap using Doppler ultrasonography guidance. An osteoplastic occipital craniotomy was performed and the dura was incised (Fig. 2C) and then inverted and inserted on the brain surface around the bone window. The external surface of the dura was attached to the brain surface as an indirect revascularization. An end-to-side OA-PCA anastomosis was then performed using 10-0 monofilament nylon sutures (Fig. 2D). After anastomosis, the patency was checked visually by indocyanine green intraoperative angiography. The periosteal flap was attached to the brain surface and connected to the cut end of the dura by suturing as indirect revascularization. Two frontal burr holes measuring $1 \mathrm{~cm}$ in diameter were then made in the medial frontal region of each hemisphere under a new linear
4 months after

On set

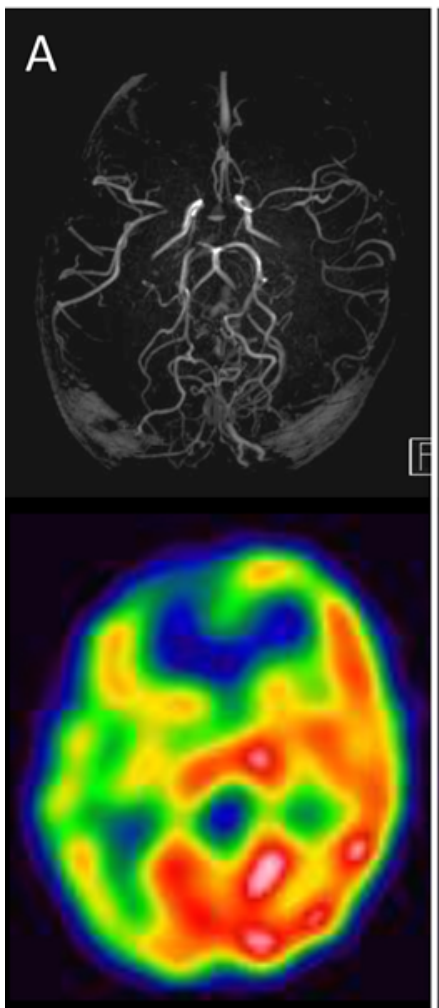

Bil. anterior revascularization

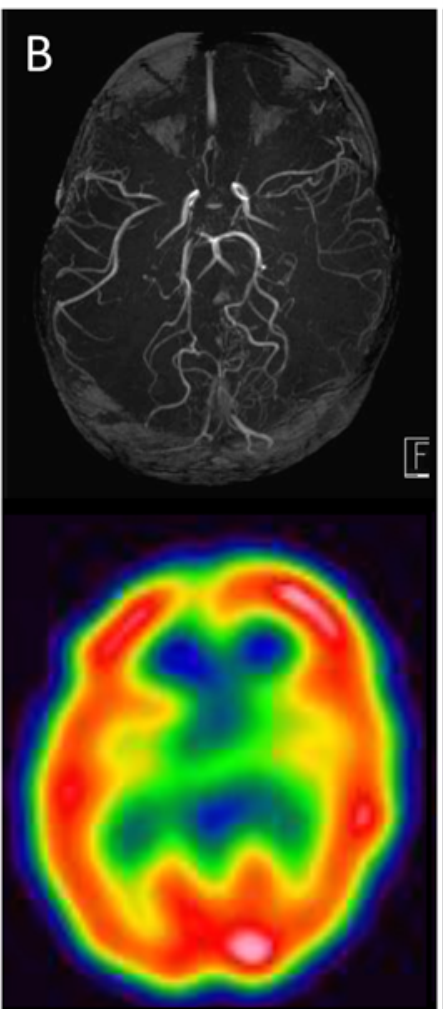

3 years after

Bil. anterior revascularization

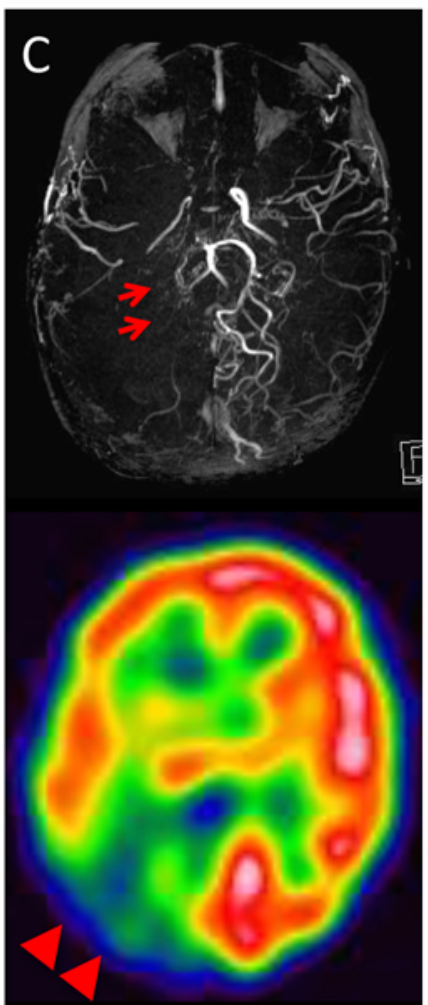

4 months after

Rt. posterior revascularization

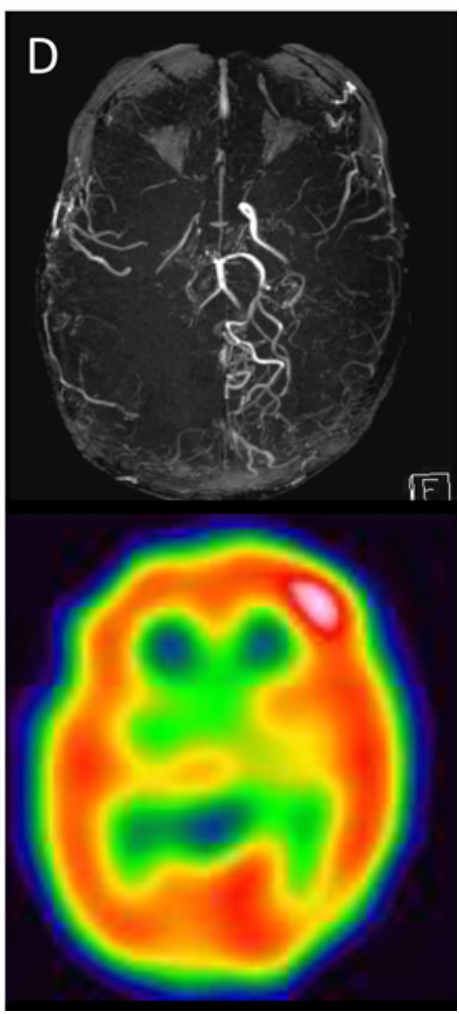

FIG. 1. Magnetic resonance angiography (upper row) and basal ${ }^{123}$ |-IMP-SPECT scan (lower row) performed at rest at the time of the initial diagnosis (A), 4 months after bilateral anterior circulation revascularization surgery (B), 3 years after bilateral anterior circulation revascularization surgery (C), and 4 months after right posterior circulation revascularization surgery (D). A markedly increased CBF following anterior and posterior revascularization was found on ${ }^{123}$-IMP-SPECT. Progressive right PCA stenosis (arrows) and decreased CBF (arrowheads) are indicated in panel C. Bil = bilateral. Figure is available in color online only. 

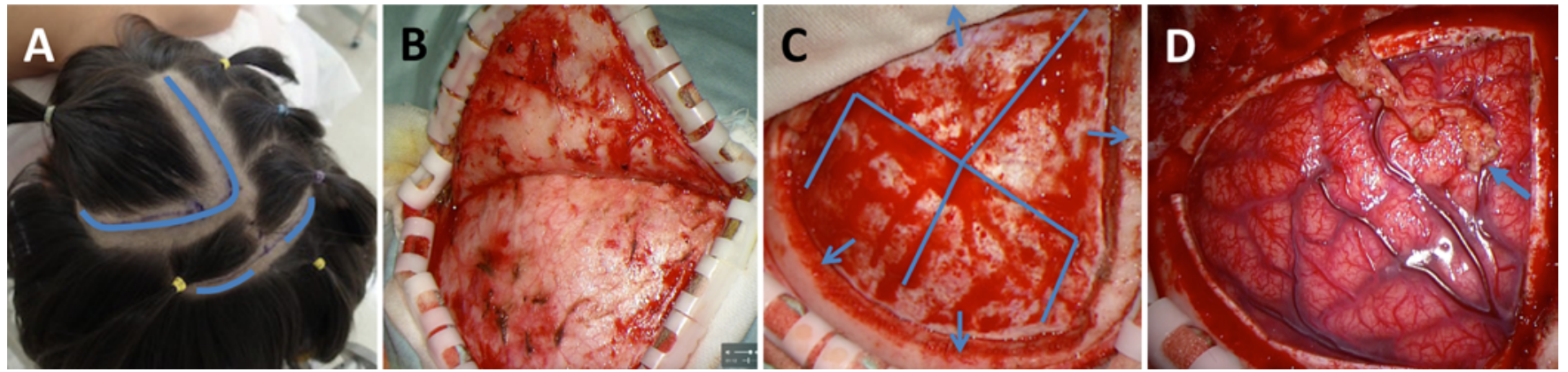

FIG. 2. Intraoperative views of OA-PCA anastomosis with indirect revascularization. A: The patient was placed prone on a horseshoe-shaped headrest. Skin incisions are indicated by blue lines. B: A reversed U-shaped occipital skin incision was made; the periosteum was left in place on the bone and later dissected to create a periosteal flap. C: An osteoplastic craniotomy was performed and the dura was incised (blue lines) and inserted on the brain surface (arrows). D: End-to-side OA-PCA anastomosis performed using 10-0 monofilament nylon sutures. An anastomosed point is indicated by the arrow. Figure is available in color online only.

skin incision. The dura mater was opened through each burr hole, with implantation of fat tissue, derived from the patient's hip, as an autograft to promote indirect revascularization. Our procedure has been described in detail in a previous report. ${ }^{3}$

\section{Postoperative Course}

Perioperative management was performed in the same manner as that for anterior revascularization. The postoperative course was uneventful, and the patient's TIA disappeared after posterior revascularization. The ${ }^{123}$ I-IMPSPECT performed at rest 4 months after surgery showed increased CBF, while MRI studies demonstrated no additional ischemic lesions (Fig. 1D).

\section{Discussion}

The results of the present study revealed a strong correlation between the presence of infarction and PCA stenosis at onset. In addition, younger age and the presence of infarction at the time of initial diagnosis were risk factors for progressive PCA stenosis after anterior revascularization. Furthermore, posterior revascularization surgery can be effective for the prevention of ischemic events for pediatric patients with MMD and posterior circulation insufficiency. Thus, we should be aware of the possibility of progressive PCA stenosis during the postoperative followup period and consider performing posterior circulation revascularization surgery, especially in patients with these risk factors.

\section{MMD and PCA Stenosis}

In this study, 23 (37\%) of 62 patients presented with PCA stenosis at the time of the initial MRA. A reported $21.7 \%-59.8 \%$ of patients with MMD present with PCA stenosis at the time of the initial diagnosis., ${ }^{2,410,20,22,23} \mathrm{Re}$ cent studies reported the insufficiency of posterior circulation in patients with MMD as an important factor related to poor prognosis. Especially in patients with the homozygous c. $14576 \mathrm{G}>\mathrm{A}$ variant of the ring finger protein 213 , infarction at initial presentation and PCA involvement are significantly more frequent. ${ }^{22}$ Collateral flow through the PCA as a leptomeningeal feeder develops to supply blood flow to the ischemic brain of the anterior circulation in patients with MMD. In other words, as PCA stenosis progresses, the cerebral perfusion pressure (CPP) to the ischemic brain of the anterior circulation through the leptomeningeal collaterals decreases, resulting in infarction. Thus, revascularization surgery should be performed as soon as possible if a patient shows PCA stenosis at onset to prevent further cerebral infarction.

\section{Progression of PCA Stenosis After Anterior Revascularization}

The results of this study indicate that younger age at onset and the presence of infarction at the time of the initial diagnosis are significant risk factors for progressive PCA stenosis after anterior revascularization. The progression of ICA and/or PCA stenosis in MMD after anterior circulation revascularization surgery is not rare; instead, it is expected. However, there are few reports regarding the factors related to the progression of PCA stenosis after anterior revascularization. Huang et al. ${ }^{6}$ reported progression of angiographic ICA stenosis in 55.2\%, PCA stenosis in $47.4 \%$, and symptomatic PCA stenosis in $21.0 \%$ of 23 patients during a minimum follow-up period of 3 years after anterior circulation revascularization surgery. They found 2 risk factors of more advanced preoperative ICA stage and the presence of stenoocclusive disease involving the PCA on preoperative angiography. In addition, Lee et al. ${ }^{13}$ showed that the presence of initial infarction and a smaller angle between the PCA and basilar artery were associated with progressive stenosis of PCA after anterior revascularization. We hope to increase awareness of these risk factors for the management of MMD to prevent further cerebral infarctions and protect the developing brain.

\section{Symptoms Due to Progressive PCA Stenosis After Anterior Revascularization}

In our study, 9 of 12 patients with progressive PCA stenosis after anterior revascularization demonstrated ischemic symptoms, such as transient lower-limb paresis or decreased uptake on ${ }^{123}$ I-IMP-SPECT, and required 
posterior circulation revascularization surgery. In general, lower-limb paresis is not a PCA-specific symptom, such as visual field defect, but rather an ischemic symptom related to the ACA territory. We propose 2 explanations for the leg symptoms. The first explanation is that the CPP of the medial part of the precentral area through the leptomeningeal collaterals from the PCA territory decreases as PCA stenosis progresses because the posterior pericallosal arteries also supply the medial part of the precentral area. As a result, new ischemic symptoms, such as lower-limb paresis, may develop. Furthermore, because the ACA territory may not be easily addressed on the initial anterior revascularization surgery, the CPP of the medial part of the precentral cortex may be prone to decrease after anterior revascularization with PCA progression. To resolve this problem, we create a craniotomy as large as possible during anterior revascularization to promote neovascularization and successful indirect revascularization; however, the ACA territory, such as the precentral cortex, can demonstrate poor neovascularization. An alternative explanation is difficulties in detecting transient visual disturbances in young children because they cannot clearly describe their symptoms. Our hospital is customized for young children in our district; most of our patients are babies, toddlers, and early elementary school children. Lee et al. ${ }^{12}$ also analyzed 41 pediatric patients with MMD, reporting that headache was the most common symptom (n $=32$, followed by transient visual symptoms $(\mathrm{n}=22)$ due to postoperative PCA stenosis. Furthermore, Kazumata et al. ${ }^{10}$ assessed 3 adults and 7 pediatric patients with posterior revascularization, reporting symptoms due to progressive PCA stenosis, including transient motor deficits involving the lower extremities $(\mathrm{n}=5)$, visual disturbances $(n=6)$, and cerebral infarctions $(n=6)$. Therefore, the symptoms due to progressive PCA stenosis after anterior revascularization are not always PCA specific. Technical differences in the initial operative procedures for the anterior circulation may also be associated with differences in symptoms due to PCA stenosis and/or time intervals after anterior revascularization. Kuroda et al. ${ }^{11}$ described a surgical procedure to revascularize the medial frontal lobe using the frontal pericranial flap through a medial frontal craniotomy in addition to conventional STA-MCA anastomosis and indirect bypass. If the ACA territory including the precentral cortex is well addressed in the initial surgery, the most common symptoms might change and posterior revascularization may not be necessary. Further development of the operative procedure is always needed.

\section{Posterior Revascularization Surgery}

PCA involvement in patients with MMD was first reported in the $1980 \mathrm{~s} ;{ }^{17-21}$ however, few reports have assessed posterior circulation revascularization surgery. The reported rates of posterior revascularization after anterior revascularization in pediatric patients with MMD are $10.3 \%-12.2 \%{ }^{1,12}$ In our study, $14.5 \%$ (9/62 pediatric patients) required posterior revascularization. This rate may depend on the initial surgical procedures, but approximately $10 \%-15 \%$ of pediatric patients with MMD will require posterior revascularization after anterior revascularization. In 1986, Miyamoto et al. ${ }^{18}$ first described indi- rect revascularization surgery using an omental flap transplantation to the occipital lobe in 5 patients with MMD, including 4 children. They reported that 34 (26.4\%) of 129 pediatric patients with MMD had several types of visual disturbances due to occlusive lesions in the posterior circulation and emphasized the importance of posterior revascularization. Lee et al..$^{12}$ also reported that $41(12.2 \%)$ of 335 pediatric patients with MMD underwent indirect posterior revascularization surgery, including EDAS and multiple burr hole trephinations that improved symptoms and radiological findings. Recently, Kazumata et al..$^{10}$ also reported OA-PCA anastomosis with an indirect bypass in 10 patients $(3.5 \%)$ with MMD, including 7 pediatric patients, after anterior revascularization. Their report included both adults and children, so their results may show a lower rate of posterior revascularization. Furthermore, they simultaneously performed bilateral posterior revascularization in 3 of the 7 pediatric patients. We also chose OA-PCA anastomosis with indirect revascularization as an operative procedure for posterior circulation, ${ }^{3}$ except that we could not find optimal recipient arteries to be anastomosed on the brain surface. We also performed bilateral posterior revascularization in 2 of 9 patients (cases 3 and 7). Bilateral simultaneous posterior revascularization is necessary for certain patients. It is slightly more difficult to dissect the OA than the STA. However, because of the quick improvement of the CBF immediately after surgery, we believe it to be more effective for pediatric patients with progressive MMD compared with the use of indirect procedures only. In addition, we add burr hole procedures in the medial frontal region with implantation of fat tissue derived from the patient's hip. For patients with posterior revascularization, this can be the last revascularization, so indirect revascularization should be designed as much as possible.

Surgical indication for additional revascularization in the posterior portion of the brain remains controversial because it is difficult to detect and clarify symptoms due to PCA stenosis, especially in young children. Generally, anterior revascularization was the first treatment choice at the time of diagnosis, regardless of PCA involvement. Patients demonstrating ischemic symptoms attributable to PCA lesions after anterior revascularization are optimal candidates. However, because of the progressive nature of MMD, we should consider posterior revascularization surgery even in asymptomatic patients if they show progressive PCA stenosis and decreased uptake on ${ }^{123}$ I-IMPSPECT in the posterior circulation to prevent cerebral infarctions.

\section{Conclusions}

PCA stenosis in MMD is an important clinical factor related to poor prognosis before and after anterior circulation revascularization surgery. Younger age at onset and the presence of infarction at the time of the initial diagnosis are significant risk factors for progressive PCA stenosis after anterior revascularization. Posterior circulation revascularization surgery, including OA-PCA anastomosis, can be effective. We should be aware of the possibility of progressive PCA stenosis during the postoperative follow- 
up period and consider performing posterior circulation revascularization surgery.

\section{References}

1. Funaki T, Takahashi JC, Takagi Y, Yoshida K, Araki Y, Kikuchi T, et al: Impact of posterior cerebral artery involvement on long-term clinical and social outcome of pediatric moyamoya disease. J Neurosurg Pediatr 12:626-632, 2013

2. Funaki T, Takahashi JC, Takagi Y, Yoshida K, Kikuchi T, Mineharu Y, et al: [Revascularization for posterior cerebral artery territory in pediatric moyamoya disease], presented at the 76th Annual Meeting of the Japan Neurosurgical Society. Tokyo: Japan Neurosurgical Society, 2017 (Abstract) (Jpn)

3. Hayashi T, Shirane R, Tominaga T: Additional surgery for postoperative ischemic symptoms in patients with moyamoya disease: the effectiveness of occipital artery-posterior cerebral artery bypass with an indirect procedure: technical case report. Neurosurgery 64:E195-E196, 2009

4. Hishikawa T, Tokunaga K, Sugiu K, Date I: Assessment of the difference in posterior circulation involvement between pediatric and adult patients with moyamoya disease. J Neurosurg 119:961-965, 2013

5. Hishikawa T, Tokunaga K, Sugiu K, Date I: Long-term outcomes in adult patients with ischemic-type moyamoya disease involving posterior circulation. Acta Neurochir (Wien) 156:1745-1751, 2014

6. Huang AP, Liu HM, Lai DM, Yang CC, Tsai YH, Wang KC, et al: Clinical significance of posterior circulation changes after revascularization in patients with moyamoya disease. Cerebrovasc Dis 28:247-257, 2009

7. Huang AP, Tu YK: Progressive PCA steno-occlusive changes after revascularization for moyamoya disease: a neglected phenomenon. Neurosurgery 67:E1865-E1866, 2010 (Letter)

8. Karasawa J, Kikuchi H, Furuse S, Kawamura J, Sakaki T: Treatment of moyamoya disease with STA-MCA anastomosis. J Neurosurg 49:679-688, 1978

9. Karasawa J, Kikuchi H, Furuse S, Sakaki T, Yoshida Y: A surgical treatment of "moyamoya" disease "encephalo-myo synangiosis". Neurol Med Chir (Tokyo) 17:29-37, 1977

10. Kazumata K, Kamiyama H, Saito H, Maruichi K, Ito M, Uchino H, et al: Direct anastomosis using occipital artery for additional revascularization in moyamoya disease after combined superficial temporal artery-middle cerebral artery and indirect bypass. Oper Neurosurg (Hagerstown) 13:213-223, 2017

11. Kuroda S, Houkin K, Ishikawa T, Nakayama N, Iwasaki Y: Novel bypass surgery for moyamoya disease using pericranial flap: its impacts on cerebral hemodynamics and long-term outcome. Neurosurgery 66:1093-1101, 2010

12. Lee JY, Choi YH, Cheon JE, Paeng JC, Ryu HW, Kim KJ, et al: Delayed posterior circulation insufficiency in pediatric moyamoya disease. J Neurol 261:2305-2313, 2014

13. Lee JY, Kim SK, Cheon JE, Choi JW, Phi JH, Kim IO, et al: Posterior cerebral artery involvement in moyamoya disease: initial infarction and angle between PCA and basilar artery. Childs Nerv Syst 29:2263-2269, 2013

14. Matsushima Y, Inaba Y: The specificity of the collaterals to the brain through the study and surgical treatment of moyamoya disease. Stroke 17:117-122, 1986

15. Matsushima Y, Suzuki R, Yamaguchi T, Tabata H, Inaba Y: [Effects of indirect EC/IC bypass operations on adult moyamoya patients.] No Shinkei Geka 14:1559-1566, 1986 (Jpn)

16. Matsushima Y, Yamaguchi T, Takasato Y, Tomida S, Fukumoto T, Suzuki R, et al: [Changes in symptoms after enceph- alo-duro-arterio-synangiosis (EDAS) in pediatric moyamoya disease.] No To Hattatsu 18:3-7, 1986 (Jpn)

17. Miyamoto S, Kikuchi H, Karasawa J, Nagata I: [Visual disturbances in moyamoya disease.] No To Shinkei 38:765-772, 1986 (Jpn)

18. Miyamoto S, Kikuchi H, Karasawa J, Nagata I, Ihara I, Yamagata S: Study of the posterior circulation in moyamoya disease. Part 2: Visual disturbances and surgical treatment. J Neurosurg 65:454-460, 1986

19. Miyamoto S, Kikuchi H, Karasawa J, Nagata I, Ikota T: Moyamoya disease associated with bilateral occlusion of the vertebral artery. Surg Neurol 22:21-28, 1984

20. Miyamoto S, Kikuchi H, Karasawa J, Nagata I, Ikota T, Takeuchi S: Study of the posterior circulation in moyamoya disease. Clinical and neuroradiological evaluation. J Neurosurg 61:1032-1037, 1984

21. Miyamoto S, Kikuchi H, Karasawa J, Nagata I, Ikota T, Takeuchi S: [Study on the vertebro-basilar system in "moyamoya" disease.] No To Shinkei 36:491-499, 1984 (Jpn)

22. Miyatake S, Miyake N, Touho H, Nishimura-Tadaki A, Kondo Y, Okada I, et al: Homozygous c. $14576 \mathrm{G}>\mathrm{A}$ variant of RNF213 predicts early-onset and severe form of moyamoya disease. Neurology 78:803-810, 2012

23. Mugikura S, Higano S, Shirane R, Fujimura M, Shimanuki Y, Takahashi S: Posterior circulation and high prevalence of ischemic stroke among young pediatric patients with moyamoya disease: evidence of angiography-based differences by age at diagnosis. AJNR Am J Neuroradiol 32:192-198, 2011

24. Mugikura S, Takahashi S: Posterior circulation in moyamoya. J Neurosurg 120:1240-1241, 2014

25. Research Committee on the Pathology and Treatment of Spontaneous Occlusion of the Circle of Willis: Guidelines for diagnosis and treatment of moyamoya disease (spontaneous occlusion of the circle of Willis). Neurol Med Chir (Tokyo) 52:245-266, 2012

26. Suzuki J, Takaku A: Cerebrovascular "moyamoya" disease. Disease showing abnormal net-like vessels in base of brain. Arch Neurol 20:288-299, 1969

\section{Disclosures}

The authors report no conflict of interest concerning the materials or methods used in this study or the findings specified in this paper.

\section{Author Contributions}

Conception and design: Kimiwada. Acquisition of data: Kimiwada. Analysis and interpretation of data: Kimiwada. Drafting the article: Kimiwada. Critically revising the article: Kimiwada, Hayashi. Reviewed submitted version of manuscript: Kimiwada. Approved the final version of the manuscript on behalf of all authors: Kimiwada. Statistical analysis: Kimiwada. Study supervision: Hayashi, Shirane, Tominaga.

\section{Supplemental Information Previous Presentations}

The abstract and parts of the text were presented at the 44th Annual Meeting of the International Society for Pediatric Neurosurgery, in Kobe, Japan, October 23-27, 2016.

\section{Correspondence}

Tomomi Kimiwada: Miyagi Children's Hospital, Sendai, Japan. tomomi@nsg.med.tohoku.ac.jp. 